\title{
Intrathecal insulin-like growth factor 1 but not insulin enhances myelin repair in young and aged rats
}

Martin Hlavica ${ }^{1,} 4^{*}$, Aro Delparente ${ }^{1 *}$, Andrin Good $^{1}$, Nicolas Good $^{1}$, Patricia S. Plattner ${ }^{1}$, Maryam S. Seyedsadr ${ }^{1}$, Martin E. Schwab ${ }^{1}$, Dianne P. Figlewicz ${ }^{2}$, Benjamin V. Ineichen $^{1,3 \#}$

${ }^{1}$ Brain Research Institute, University of Zurich, and Department of Health Sciences and Technology,

ETH Zurich, 8057 Zurich, Switzerland

${ }^{2}$ VA Puget Sound Health Care System and University of Washington, Seattle, Washington, U.S.A.

${ }^{3}$ University Hospital Zurich, Department of Neurology, 8091 Zurich, Switzerland

${ }^{4}$ Cantonal Hospital St.Gallen, Department of Neurosurgery, Switzerland

*These authors contributed equally and share the first authorship

Running title: Insulin-like growth factor-1 but not insulin enhances myelin repair

Word count

In the abstract: 133

In the manuscript: 2351

Number of figures: 5

"Corresponding author

Benjamin V. Ineichen

E-Mail: ineichen@protonmail.ch

Phone: +4144635 3225 


\begin{abstract}
One main pathological hallmark of multiple sclerosis (MS) is demyelination. Novel therapies which enhance myelin repair are urgently needed. Insulin and insulin-like growth factor 1 (IGF-1) have strong functional relationships. Here, we addressed the potential capacity of IGF-1 and insulin to enhance remyelination in an animal demyelination model in vivo. We found that chronic intrathecal infusion of IGF-1 enhanced remyelination after lysolecithininduced demyelination in the spinal cord of young and aged rats. Aged rats showed a weaker innate remyelination capacity and are therefore a good model for progressive MS which is defined by chronic demyelination. In contrast to IGF-1, Insulin had no effect on remyelination in either age group. Our findings highlight the potential use of IGF-1 as remyelinating therapy for MS, particularly the progressive stage in which chronic demyelination is the hallmark.
\end{abstract}

\title{
Keywords
}

Multiple Sclerosis, lysolecithin, IGF-1, insulin, remyelination, rat, intrathecal

\section{Highlights}

Insulin-like growth factor 1 (IGF-1) enhanced remyelination in young and aged rats.

Insulin, functionally related to IGF-1, did not enhance remyelination.

Aged rats show less pronounced myelin repair compared to young rats.

Aged rats are model for chronic demyelination faced in chronic multiple sclerosis. 


\section{Introduction}

Multiple sclerosis (MS) is a disease with multifactorial etiology and both genetic and environmental factors contribute to the risk of the disease [10]. Pathological hallmarks of this neuro-inflammatory disease are immune cell infiltration into the central nervous system (CNS) parenchyma, demyelination, and neurodegeneration [28]. Some treatment options are currently available for the early relapsing-remitting disease stage of MS which act mostly through modulation of the immune system. However, only a small number of therapies exist for the chronic progressive disease stage in which demyelination and neurodegeneration are pathological hallmarks [25]. Therefore, finding therapies that potentially restore the myelin sheath, re-establish efficient axonal conduction, and protect axons from secondary neurodegeneration has high priority in neurological research[8].

A recent study has re-invigorated interest in insulin-like growth factor 1 (IGF-1) as a potential therapy for neuro-inflammation [1]. Whereas IGF-1 was shown to be a potent promotor of developmental myelin formation [33], its potential to enhance remyelination remained controversial $[6,27,32]$. The functionally and structurally related hormone insulin could yet be another approach to boost remyelination. It shows some crossreactivity at the level of receptors, shares some signaling pathways with IGF-1 $[21,30]$ and has been shown to enhance remyelination in vitro [16, 29].

Here, we used lysolecithin injections into the spinal cord dorsal funiculi as a model of local demyelination, and compared the effects of chronic intrathecal infusion of IGF-1 or insulin on myelin repair in young and aged rats. Aged rats show weak innate remyelination and are therefore a good model for chronic demyelination, similar to the situation in progressive MS [24]. 


\section{Materials and methods}

\section{Animals}

Young (12 - 14 weeks, $200-300$ g) and aged (12 months, $300-400$ gram) female Long Evans rats were used for all experiments (LE, strain code 006, Charles River, Italy). Rats were housed in a specific-pathogen-free (SPF) animal facility in groups of $3-4$ rats under a constant $12 \mathrm{~h}$ light/dark cycle (light from 06:00am to 06:00 pm) with standard rodent chow and water ad libitum. All animal procedures and protocols were approved by the Veterinary Office of the Canton of Zurich, Switzerland.

\section{Lysolecithin injections}

Lysolecithin injections in the cervical spinal cord were performed as previously described [2]: briefly, rats were deeply anesthetized under fentanyl $(0.005 \mathrm{mg} / \mathrm{kg})$, medetomidine $(0.15 \mathrm{mg} / \mathrm{kg})$, and midazolam $(2 \mathrm{mg} / \mathrm{kg})$. The cervical spinal cord at spinal level $\mathrm{C} 4$ was subsequently exposed. Using a 35 gauge Hamilton syringe (World Precision Instruments, Sarasota), $2 \mu \mathrm{l}$ of a sterile $1 \%$ lysolecithin solution (Sigma, St. Louis) in phosphate-buffered saline (PBS) were slowly injected $(10 \mathrm{nl} / \mathrm{sec})$ in the midline $400 \mu \mathrm{m}$ deep targeting the dorsal funiculi. At the end of the injection, the injection cannula remained in position for 2 minutes until withdrawal. The injection position in the spinal cord was marked by applying a droplet of Monestral blue after the injection.

\section{Intrathecal insulin and IGF-1 treatment}

To ensure sufficient local bioavailability of IGF-1 and insulin, the compounds were applied intrathecally via a catheter inserted to the subdural space of the cisterna magna. This catheter was pushed over the spinal cord in caudal direction so that the catheter tip was in 
proximity to the lysolecithin lesion at spinal level C4. It was connected to a subcutaneous osmotic minipump (Alzet, model 2ML2, Germany), subcutaneously implanted on the back of the rats as previously described [19]. An experimental timeline is depicted in Figure 1.

The pump supplied the intrathecal space constantly with either $100 \mu \mathrm{g} /$ day IGF-1 (Peprotech, USA), 3 units (U)/day (= $104 \mu \mathrm{g} /$ day) insulin (Actrapid, HM Penfill), or vehicle solution over a total treatment period of 3 weeks. IGF-1 and insulin were diluted in the vehicle solution consisting of $10 \mathrm{mM}$ acetic acid, $0.1 \mathrm{M} \mathrm{NaCl}$, and $0.1 \%$ Tween, $\mathrm{pH} 5.4$ [6]. These concentrations of IGF-1 and insulin have equivalent molar concentrations. The catheters were flushed before implantation with the corresponding treatment solution in order to avoid sticking of the compounds to the catheter walls.

\section{In vivo measurements}

Since centrally applied insulin can lead to a reduction of food and water intake via the hypothalamus $[4,12,13]$, we monitored weight as well as food and water intake during night and day by weighing water bottles and chow twice daily (08:00 am and 08:00pm). To measure potential decreases in blood glucose levels by insulin reaching the periphery from the intrathecal space, blood glucose was measured once a day by sampling a small droplet of blood taken from the tip of the rat tail. Blood sugar was measured by blood glucose test sticks and the corresponding console (Freestyle Lite, Abbott, USA).

\section{Analysis of remyelination}

Series of semithin sections stained with toluidine blue were taken at the level of the lesion center (defined in the intact spinal cord under a stereomicroscope), and approximately 1 
$\mathrm{mm}$ caudally and $1 \mathrm{~mm}$ rostrally of the lesion center and analysed by light microscopy at 600x. The amount of disproportionally thin myelin compared to the axon diameter (representing remyelinated axons [2]) was quantitated in the images of these lesions within the dorsal funiculus using imageJ (NIH, USA). The sensory tracts (cuneate and gracile fascicles) of the dorsal funiculus consist mainly of large diameter axons allowing unequivocal identification of remyelinated axons. These counts were normalized to the lesion area and expressed as remyelinated axons per $1000 \mathrm{\mu m}^{2}$. One rat of the control group of aged rats had to be excluded from the analysis due to missing lysolecithin-induced demyelination. All analyses were done at 21 days after lesion induction.

\section{Quantification of macrophages within the lysolecithin lesion}

Macrophages were quantified within the lesions as cells densely packed with myelin debris according to standard morphological criteria [22]. The cell counts were normalized to the $\underline{\text { total lesion area. All analyses were done at } 21 \text { days after lesion induction. }}$

\section{Statistical analysis/experimental design}

In all animal experiments, experimenters were blinded to the treatment and animals were randomly allocated to the treatment groups. Statistical analyses were carried out with Graphpad Prism version 6 (GraphPad software, USA). For comparison of blood glucose levels over time, a two-way ANOVA was performed followed by a Bonferroni post hoc test. For comparison of remyelination and macrophage count of the three treatment groups a one-way ANOVA was performed followed by a Bonferroni post hoc test. All data are shown as mean \pm standard deviation (SD). Asterisks indicate statistical significance: ${ }^{*}<0.05, * *<$ $0.01, * * *<0.001$ 



\section{Results}

Intrathecal IGF-1 or insulin did not alter food intake in rats.

The lesions induced by our stereotaxic injections of lysolecithin where entirely located within the dorsal funiculi of the spinal cord. It has been shown before that low doses $(7.5-10$ $\mathrm{mU} /$ day) of intraventricular applied insulin reduce food intake and weight of rats $[4,12,13]$. Therefore, we monitored food intake of rats during the experiment. Neither IGF-1 at 100 $\mu \mathrm{g} /$ day nor insulin at $3 \mathrm{U} /$ day altered the amount of chow eaten (Fig. 2 A, B), water intake (data not shown), or body weight (data not shown) in young or aged rats in the present study. Young rats had a higher mean food intake per 24 hours compared to aged rats (mean food intake (SD): IGF-1 group - young rats $16.2( \pm 2.2) \mathrm{g} / 24$ hours, aged rats $13.5( \pm 1.4) \mathrm{g} / 24$ hours; insulin group - young rats $18.0( \pm 2.6) \mathrm{g} / 24$ hours, aged rats $13.4( \pm 1.5) \mathrm{g} / 24$ hours; control group - young rats $16.3( \pm 3.0) \mathrm{g} / 24$ hours, aged rats $12.5( \pm 2.2) \mathrm{g} / 24$ hours).

\section{Continuous intrathecal infusion of insulin but not IGF-1 led to a decrease in blood glucose} levels.

Baseline blood glucose levels were similar in all experimental groups of young and aged rats (mean blood glucose levels (SD): IGF-1 group - young rats $6.5 \mathrm{mM}( \pm 0.29)$, aged rats $6.6 \mathrm{mM}$ $( \pm 0.29)$; insulin group - young rats $5.9( \pm 0.59)$, aged rats $6.5 \mathrm{mM}( \pm 0.26)$; control group young rats $6.1 \mathrm{mM}( \pm 0.38)$, aged rats $6.6 \mathrm{mM}( \pm 0.67))$. Rats of both age groups showed a drop in peripheral blood glucose after 1-2 days of infusion of insulin to the intrathecal space. This drop was statistically significant and different from the IGF-1 and control group up to day 9 after lesion induction and pump implantation (Fig. 3 A, B). This confirmed the in vivo viability of the insulin administration. 
Continuous intrathecal infusion of IGF-1 but not insulin enhanced remyelination of lysolecithin lesions in the spinal cord of young and aged rats.

IGF-1 infused at $100 \mu \mathrm{g} /$ day over three weeks over the lesion site intrathecally immediately following a lysolecithin injection into the dorsal funiculi enhanced remyelination in both age groups as assessed 21 days after the lysolecithin-induced local demyelination approximately by three-fold in young rats and five-fold in aged rats (count of remyelinated axons per 1000 $\mu \mathrm{m}^{2}(\mathrm{SD})$ : young rats (Fig. 4A) - IGF-1 group $18.7( \pm 7.9)$, control group $7.1( \pm 1.7)$; aged rats (Fig. 4B) - IGF-1 group $7.9( \pm 3.6)$, control group $1.6( \pm 0.2))$. Interestingly, aged rats from all treatment groups showed a much lower remyelination capacity compared to the corresponding treatment groups of young rats at 21 days after lesion induction. In sharp contrast to IGF-1, equimolar concentrations of intrathecal insulin did not change the number of remyelinated axons as compared to the vehicle treated controls (insulin group young rats $6.4( \pm 1.2)$, aged rats: $1.4( \pm 0.7)$, Fig. 4$)$.

Neither IGF-1 nor insulin did alter the amount of macrophages in the demyelinated lesion As it has been shown in the neuro-inflammatory MS animal model experimental autoimmune encephalomyelitis (EAE), IGF-1 had immune-modulatory effects dampening the disease severity $[1,9]$. To address a potential immune-modulatory effect of intrathecal IGF-1 or insulin in the lysolecithin demyelination model, we quantified myelin-debris loaded macrophages within the lesions from all groups. There was no difference in the macrophage count between IGF-1-, insulin-, or control-treated young rats (macrophages per $1000 \mu \mathrm{m}^{2}$ within the lesion (SD): IGF-1 0.54 (0.07), insulin 0.52 (0.09), control $0.57(0.08)$, Fig. 5A) or 
aged rats (macrophages per $1000 \mu \mathrm{m}^{2}$ within the lesion (SD): IGF-1 $0.29(0.06)$, insulin 0.31 (0.09), control $0.28(0.02)$ (Fig. 5B). 


\section{Discussion}

We investigated the role of the hormonal growth factors IGF-1 and insulin as potential remyelinating agents. Continuous intrathecal infusion of IGF-1 but not insulin promoted remyelination in both young and aged rats after induction of focal demyelination in the spinal cord.

It has been shown earlier that chronic intraventricular infusion of insulin at $7.5-10 \mathrm{mU} /$ day causes a reduction in food intake and body weight [4]. We therefore monitored food intake and weight in rats chronically infused with a higher insulin dose to the intrathecal space (3 $\mathrm{U} /$ day). We did not observe any impact on food intake or weight upon infusion. Inefficient distribution of insulin against the bulk flow of cerebrospinal fluid (CSF) [23] coupled with its short half-life $[5,26]$ could be responsible for intrathecal insulin not reaching the third ventricle and triggering a reduction in food intake/weight. However, insulin induced a drop in blood glucose in our study, probably by leakage to the periphery via the CSF draining system, confirming its in vivo bioactivity.

Overexpression of IGF-1 in astrocytes leads to a developmental enhancement of myelin formation [33]. However, the role of IGF-1 in remyelination after myelin damage remained controversial: treatment with IGF-1 led to an upregulation of myelin proteins in EAE, a neuro-inflammatory MS animal model [32], and local upregulation of IGF-1 mRNA using viral vectors was shown to boost remyelination after lysolecithin-induced demyelination [27]. However, another study did not confirm the latter effect in EAE [6]. Our data show a clear and pronounced remyelination-promoting effect of IGF-1 after continuous intrathecal application over 3 weeks following lysolecithin-induced demyelination. In comparison with $E A E$, toxin-induced demyelination models are more suitable to study remyelination due to 
their clear temporal separation of demyelination and subsequent myelin repair [11]. Moreover, the myelin repair is not superimposed by complex inflammatory processes.

Interestingly, aged rats showed an approximately five-fold increase in remyelination compared to young rats with a three-fold increase compared with the corresponding control group. It has already been shown that this could be attributable to delayed changes in growth factor gene expression [18].

The therapeutic potential of IGF-1 has also been tested in a clinical small pilot study on seven MS patients [14]. Subcutaneous administration of IGF-1 was safe and well tolerated; it did, however, not alter disease course or MRI measures. This failure has been attributed to insufficient penetration of IGF-1 through the blood brain barrier (BBB) [7]. Intrathecal application of this molecule could therefore be an alternative way of administration that circumvents the BBB [19]. This is already done in clinical practice, e.g. for the antibody rituximab [3], or for baclofen to treat spasticity [15] with minimal systemic side effects.

In contrast to IGF-1, insulin did not enhance remyelination. IGF-1 and insulin exert their effects via specific IGF-1- or insulin receptors, but they also share signaling pathways and show some crossreactivity due to their sequence homologies (reviewed in [21]). The enhancement of remyelination, however, was selectively mediated by IGF-1. The presence or absence of the respective receptors on oligodendrocyte precursors or on differentiated oligodendrocytes may be a reason for this discrepancy, but needs to be analysed in detail. Macrophage counts, involved in clearance of myelin debris from the lesion [22], were not different between the treatment groups in young or ages rats suggesting no major immunemodulatory effects of IGF-1 on macrophages.

The main limitation of our proof-of-principle study is its reductionist approach: we aimed at enhancing remyelination in a single demyelinated lesion in the cervical spinal cord by 
applying IGF-1 or insulin to the CSF in proximity to the lesion. MS in contrast presents with multiple demyelinated lesions scattered along the brain-spinal cord axis. It remains to be determined whether sufficient levels of IGF-1 would be distributed along the whole CSF axis and in particular within the CNS tissue upon localized application. Furthermore, we only investigated the effects of IGF-1 and insulin in female rats. Male rats could have reacted yet differently to the IGF-1 treatment due to differences in endogenous IGF-1 levels [31] or IGF-1 receptor expression [17].

In conclusion, we found that intrathecal infusion of IGF-1 but not insulin enhanced remyelination after lysolecithin-induced demyelination. Our findings furthermore highlight that aged rats are a suitable model to study chronic demyelination which is frequently often present in patients with progressive MS. 


\section{Figure legends}

Fig. 1 Experiment timeline. After baseline measurement of food intake and blood glucose levels, both young and aged rats were subjected to a lysolecithin injection (Day 0, $2 \mu \mathrm{l}, 1 \%$ lysolecithin) causing local demyelination and subsequent remyelination in the dorsal funiculus at spinal level C4. Additionally, rats were implanted with a subcutaneous osmotic minipump connected to an intrathecal catheter placed in proximity to the lesion. This minipump continuously delivered either IGF-1 (100 $\mu \mathrm{g} /$ day), insulin ( $3 \mathrm{U} /$ day), or control solution. At day 21 , rats were sacrificed and remyelination was assessed. Food and water intake as well as blood glucose was measured on daily basis.

Fig. 2 Continuous intrathecal infusion of insulin-like growth factor 1 (IGF-1) or insulin do not alter food intake in rats. (A) Mean food intake of young rats in gram per 24 hours $(n=4$ per group). (B) Mean food intake of aged rats in gram per 24 hours ( $n=4$ per group).

Fig. 3 Continuous intrathecal infusion of insulin leads to a decrease in blood glucose. This decrease occurred within 1-2 days and was apparent in young $(A)$ and aged $(B)$ rats $(n=4$ per group). Data shown are mean \pm SD. Statistical differences were determined with two-way ANOVA. Asterisks indicate significances: ${ }^{*} p<0.05, * * * p<0.001$.

Fig. 4 Continuous intrathecal infusion of insulin-like growth factor (IGF-1) but not insulin enhances remyelination in young and aged rats. (A) IGF-1 enhances remyelination in young rats as revealed by quantitation of remyelinated axons in the lysolecithin lesion (depicted 
per $1000 \mu \mathrm{m}^{2}$, specific values can be found in the results section, $\mathrm{n}=4$ per group). (B-D) Semithin sections stained with toluidine blue demonstrate enhanced remyelination in young rats (red arrowheads) in the IGF-1 group compared to the insulin and control group. (E) IGF-1 enhances remyelination in aged rats as revealed by quantitation of remyelinated axons in the lysolecithin lesion (depicted per $1000 \mu \mathrm{m}^{2}$, specific values can be found in the results section, $n=4$ in the IGF-1 and insulin group and $n=3$ in the control group). (F-H) Semithin sections stained with toluidine blue demonstrate enhanced remyelination in aged rats (red arrowheads) in the IGF-1 group compared to the insulin and control group. White dashed line represents lesion border. $\underline{M=\text { macrophage. }}$

Fig. 5 Continuous intrathecal infusion of insulin-like growth factor (IGF-1) or insulin did not alter the count of debris-loaded macrophages within the demyelinated lesion at 21 days after experimental demyelination (A) Neither IGF-1 nor equimolar concentrations of insulin did alter the amount of debris-loaded macrophages within the lysolecithin lesion in young rats (depicted per $1000 \mu \mathrm{m}^{2}$, specific values can be found in the results section, $\mathrm{n}=4$ per group) (B) The count of debris-loaded macrophages was also not altered in aged rats by intrathecal IGF-1 or insulin treatment (depicted per $1000 \mu \mathrm{m}^{2}$, specific values can be found in the results section, $n=4$ in the IGF- 1 and insulin group and $n=3$ in the control group). Bars $\underline{\text { represent mean } \pm \text { SD. Statistical analysis was done using one-way ANOVA. Abbreviations: ns, }}$ $\underline{\text { non-significant. }}$ 


\section{Acknowledgements}

This work was supported by the Swiss MS Society, the Hartmann-Müller Foundation, Zurich, the Desirée-and-Niels-Yde Foundation (to BVI) and an MD-PhD fellowship of the Swiss National Science Foundation (No. 323530_151488, to BVI). Dianne Figlewicz Lattemann is supported by a Senior Research Career Scientist award of the Dept. of Veterans Affairs.

\section{Author contributions}

$\mathrm{BVI}$ and $\mathrm{MH}$ conceived the project; $\mathrm{BVI}, \mathrm{MH}, \mathrm{AD}, \mathrm{PSP}, \mathrm{AG}$, and NG carried out the experiments and analyzed the data; DFL and MES provided critical input on the project design and data interpretation, as well as editing of the manuscript; $\mathrm{BVI}$ and $\mathrm{MH}$ wrote the manuscript.

\section{Compliance with ethical standards}

For all studies including animals, all applicable international, national, and/or institutional guidelines for the care and use of animals were followed. All animal procedures and protocols were approved by the Veterinary Office of the Canton of Zurich, Switzerland. This article is written in accordance with the ARRIVE Guidelines [20].

\section{Conflict of interest}

All authors declare that they have no conflict of interest. Role of the sponsor: neither the Swiss National Science Foundation, the Christopher and Dana Reeve Foundation the Swiss MS Society, nor the Hartmann-Müller Foundation had a role in the design and conduct of 
the study; collection, management, analysis, and interpretation of the data; preparation, review, or approval of the manuscript; and decision to submit the manuscript for publication. The contents of this paper do not represent the views of the U.S. Department of Veterans Affairs or the United States Government. 


\section{References}

[1] D. Bilbao, L. Luciani, B. Johannesson, A. Piszczek, N. Rosenthal, Insulin-like growth factor-1 stimulates regulatory $T$ cells and suppresses autoimmune disease, EMBO molecular medicine 6 (2014) 1423-1435.

[2] W.F. Blakemore, R.J. Franklin, Remyelination in experimental models of toxin-induced demyelination, Current topics in microbiology and immunology 318 (2008) 193-212.

[3] M. Bonnan, S. Ferrari, E. Bertandeau, S. Demasles, E. Krim, M. Miquel, B. Barroso, Intrathecal rituximab therapy in multiple sclerosis: review of evidence supporting the need for future trials, Current drug targets 15 (2014) 1205-1214.

[4] D.J. Brief, J.D. Davis, Reduction of food intake and body weight by chronic intraventricular insulin infusion, Brain research bulletin 12 (1984) 571-575.

[5] X. Canas, J.A. Fernandez-Lopez, A. Ardevol, C. Adan, M. Esteve, I. Rafecas, X. Remesar, M. Alemany, Rat insulin turnover in vivo, Endocrinology 136 (1995) 3871-3876.

[6] B. Cannella, D. Pitt, E. Capello, C.S. Raine, Insulin-like growth factor-1 fails to enhance central nervous system myelin repair during autoimmune demyelination, The American journal of pathology 157 (2000) 933-943.

[7] D. Chesik, N. Wilczak, J. De Keyser, The insulin-like growth factor system in multiple sclerosis, International review of neurobiology 79 (2007) 203-226.

[8] G. Criste, B. Trapp, R. Dutta, Axonal loss in multiple sclerosis: causes and mechanisms, Handbook of clinical neurology 122 (2014) 101-113.

[9] M.F. Cusick, J.E. Libbey, N.S. Trede, R.S. Fujinami, Targeting insulin-like growth factor 1 leads to amelioration of inflammatory demyelinating disease, PloS one 9 (2014) e94486.

[10] C.A. Dendrou, L. Fugger, M.A. Friese, Immunopathology of multiple sclerosis, Nature reviews. Immunology 15 (2015) 545-558.

[11] M. Dubois-Dalcq, C. Ffrench-Constant, R.J. Franklin, Enhancing central nervous system remyelination in multiple sclerosis, Neuron 48 (2005) 9-12.

[12] D.P. Figlewicz, Adiposity signals and food reward: expanding the CNS roles of insulin and leptin, American journal of physiology. Regulatory, integrative and comparative physiology 284 (2003) R882-892.

[13] D.P. Figlewicz, S.C. Benoit, Insulin, leptin, and food reward: update 2008, American journal of physiology. Regulatory, integrative and comparative physiology 296 (2009) R9-r19.

[14] J.A. Frank, N. Richert, B. Lewis, C. Bash, T. Howard, R. Civil, R. Stone, J. Eaton, H. McFarland, T. Leist, A pilot study of recombinant insulin-like growth factor-1 in seven multiple sderosis patients, Multiple sclerosis (Houndmills, Basingstoke, England) 8 (2002) 24-29.

[15] R. Gold, C. Oreja-Guevara, Advances in the management of multiple sclerosis spasticity: multiple sclerosis spasticity guidelines, Expert review of neurotherapeutics 13 (2013) 55-59.

[16] X. Gong, Z. Xie, H. Zuo, Invivo insulin deficiency as a potential etiology for demyelinating disease, Medical hypotheses 71 (2008) 399-403.

[17] J. Hami, A. Sadr-Nabavi, M. Sankian, M. Balali-Mood, H. Haghir, The effects of maternal diabetes on expression of insulin-like growth factor-1 and insulin receptors in male developing rat hippocampus, Brain structure \& function 218 (2013) 73-84.

[18] G.L. Hinks, R.J. Franklin, Delayed changes in growth factor gene expression during slow remyelination in the CNS of aged rats, Molecular and cellular neurosciences 16 (2000) 542556.

[19] B.V. Ineichen, L. Schnell, M. Gullo, J. Kaiser, M.P. Schneider, A.C. Mosberger, N. Good, M. Linnebank, M.E. Schwab, Direct, long-term intrathecal application of therapeutics to the rodent CNS, Nature protocols 12 (2017) 104-131.

[20] C. Kilkenny, W.J. Browne, I.C. Cuthill, M. Emerson, D.G. Altman, Improving bioscience research reporting: the ARRIVE guidelines for reporting animal research, PLoS biology 8 (2010) e1000412.

[21] A. Kleinridders, Deciphering brain insulin receptor and IGF1 receptor signalling, Journal of 
neuroendocrinology (2016).

[22] M.R. Kotter, C. Zhao, N. van Rooijen, R.J. Franklin, Macrophage-depletion induced impairment of experimental CNS remyelination is associated with a reduced oligodendrocyte progenitor cell response and altered growth factor expression, Neurobiology of disease 18 (2005) 166-175.

[23] J. Kreuter, D.J. Begley, M.W. Bradbury, D.J. Begley, A. Regina, F. Roux, C. Rollinson, J. Abbott, E. Ullah Khan, The Role of Brain Extracellular Fluid Production and Efflux Mechanisms in Drug Transport to the Brain. The Blood-Brain Barrier and Drug Delivery to the CNS, CRC Press, 2000.

[24] W.W. Li, J. Penderis, C. Zhao, M. Schumacher, R.J. Franklin, Females remyelinate more efficiently than males following demyelination in the aged but not young adult CNS, Experimental neurology 202 (2006) 250-254.

[25] D.H. Mahad, B.D. Trapp, H. Lassmann, Pathological mechanisms in progressive multiple sclerosis, The Lancet. Neurology 14 (2015) 183-193.

[26] D.R. Matthews, A.S. Rudenski, M.A. Burnett, P. Darling, R.C. Turner, The half-life of endogenous insulin and C-peptide in man assessed by somatostatin suppression, Clinical endocrinology 23 (1985) 71-79.

[27] M.T. O'Leary, G.L. Hinks, H.M. Charlton, R.J. Franklin, Increasing local levels of IGF-I mRNA expression using adenoviral vectors does not alter oligodendrocyte remyelination in the CNS of aged rats, Molecular and cellular neurosciences 19 (2002) 32-42.

[28] B.F. Popescu, I. Pirko, C.F. Lucchinetti, Pathology of multiple sclerosis: where do we stand?, Continuum 19 (2013) 901-921.

[29] G.A. Roth, V.H. Jorgensen, M.B. Bornstein, Effect of insulin, proinsulin and pancreatic extract on myelination and remyelination in organotypic nerve tissue in culture, Journal of the neurological sciences 71 (1985) 339-350.

[30] K. Siddle, Signalling by insulin and IGF receptors: supporting acts and new players, Journal of molecular endocrinology 47 (2011) R1-10.

[31] N. Uysal, S. Agilkaya, A.R. Sisman, U.M. Camsari, C. Gencoglu, A. Dayi, I. Aksu, B. Baykara, S. Cingoz, M. Kiray, Exercise increases leptin levels correlated with IGF-1 in hippocampus and prefrontal cortex of adolescent male and female rats, Journal of chemical neuroanatomy 81 (2017) 27-33.

[32] D.L. Yao, X. Liu, L.D. Hudson, H.D. Webster, Insulin-like growth factor-I given subcutaneously reduces clinical deficits, decreases lesion severity and upregulates synthesis of myelin proteins in experimental autoimmune encephalomyelitis, Life sciences 58 (1996) 1301-1306.

[33] P. Ye, G.J. Popken, A. Kemper, K. McCarthy, B. Popko, A.J. D'Ercole, Astrocyte-specific overexpression of insulin-like growth factor-I promotes brain overgrowth and glial fibrillary acidic protein expression, Journal of neuroscience research 78 (2004) 472-484. 


\section{Experimental timeline}

\section{Young rats}

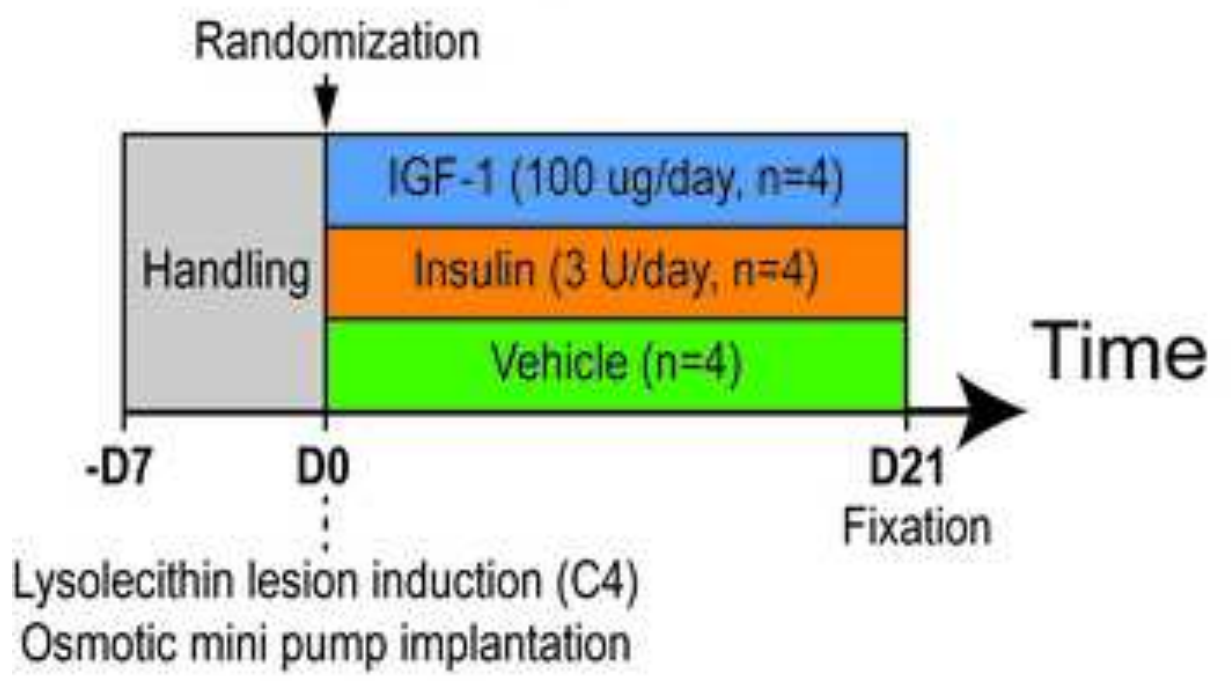

Aged rats

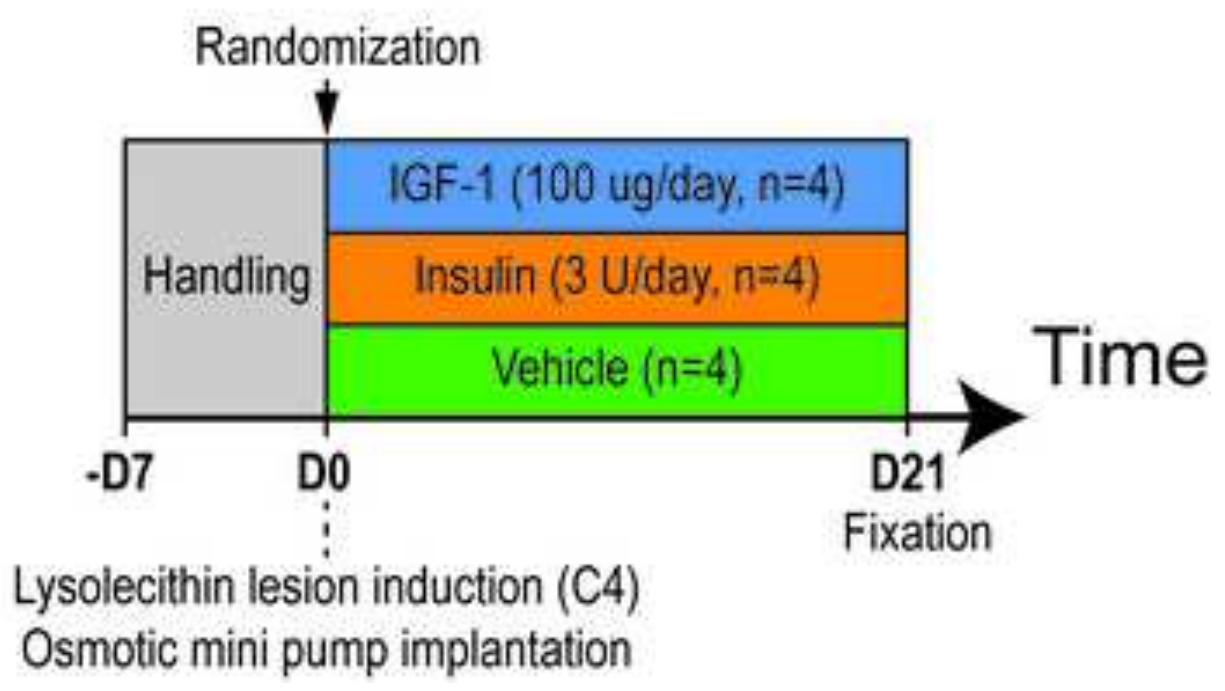



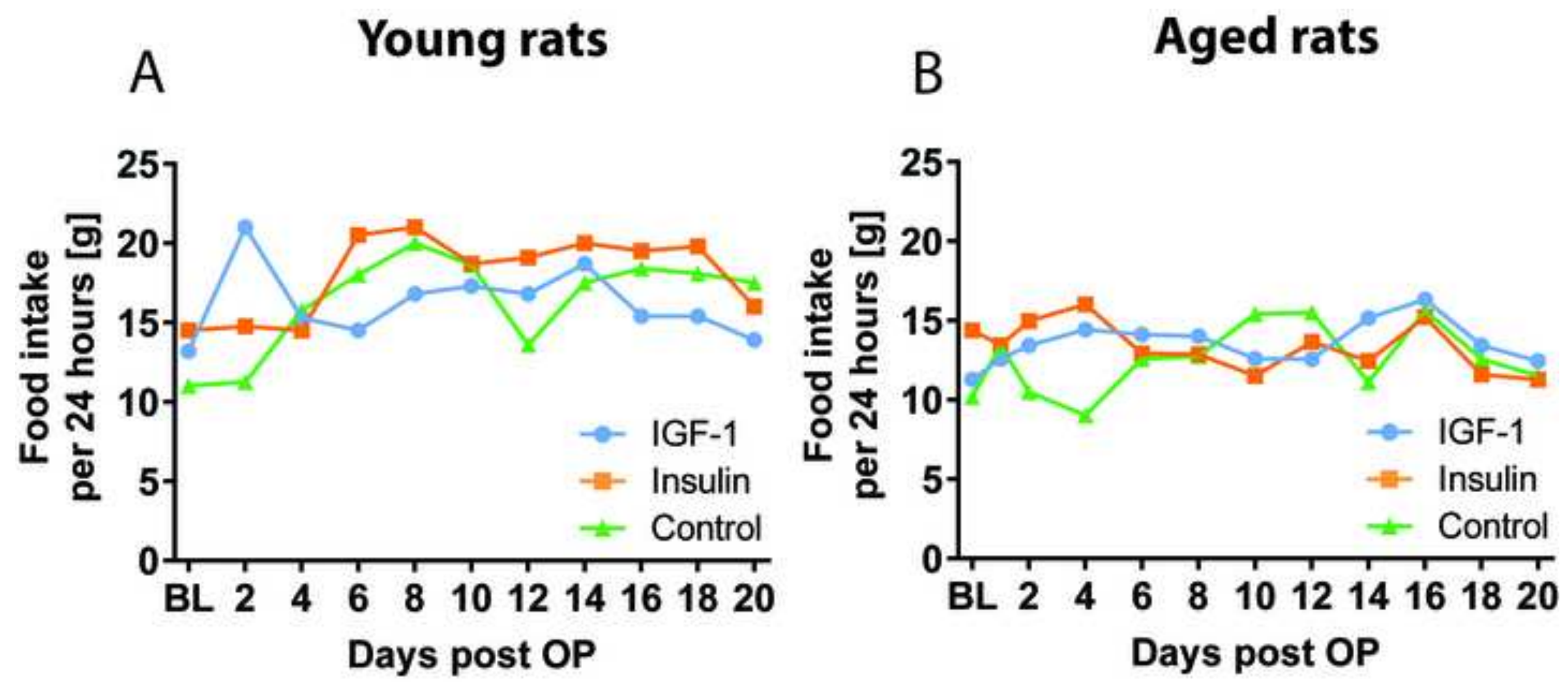
A Young rats

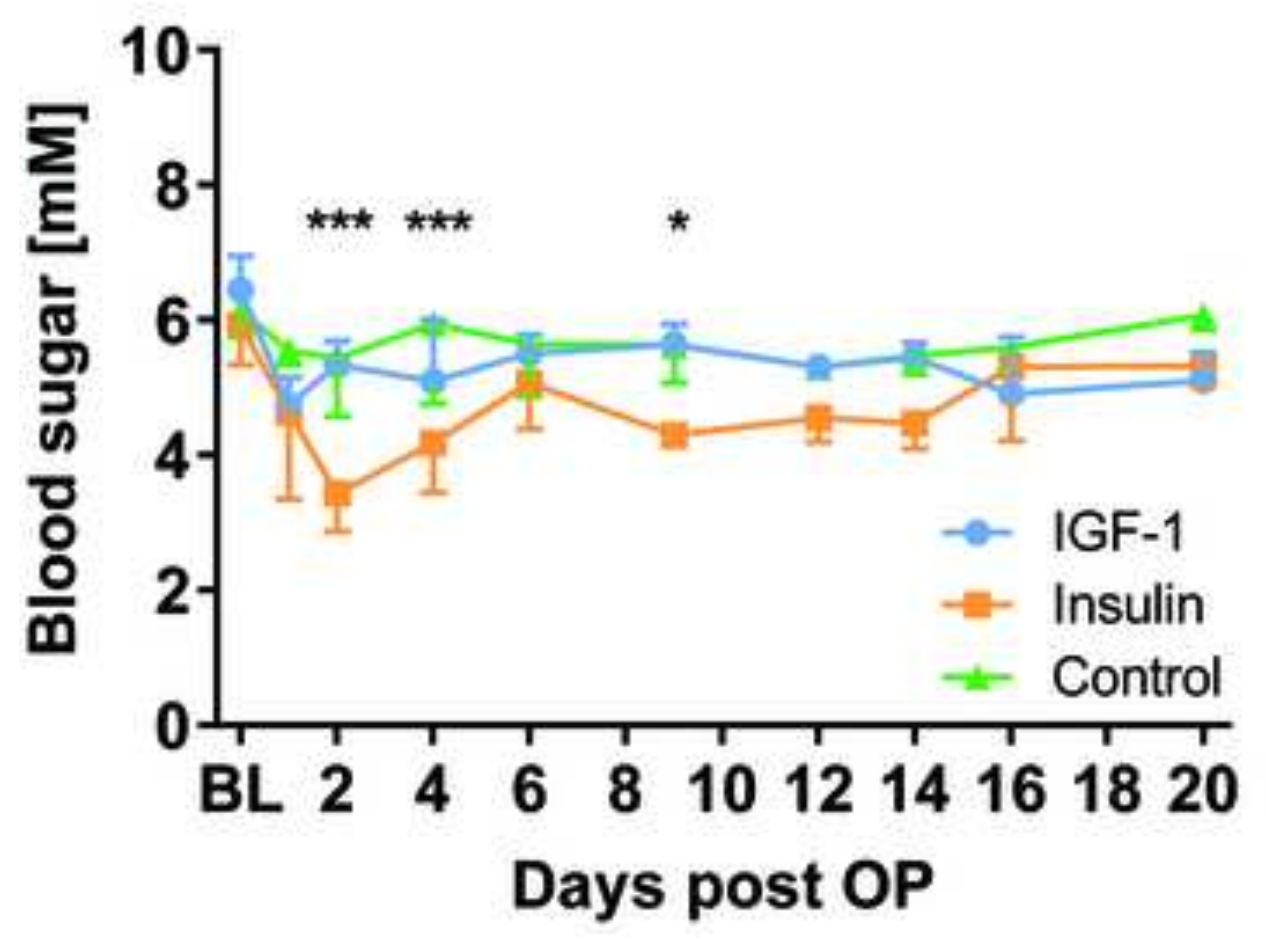

B Aged rats

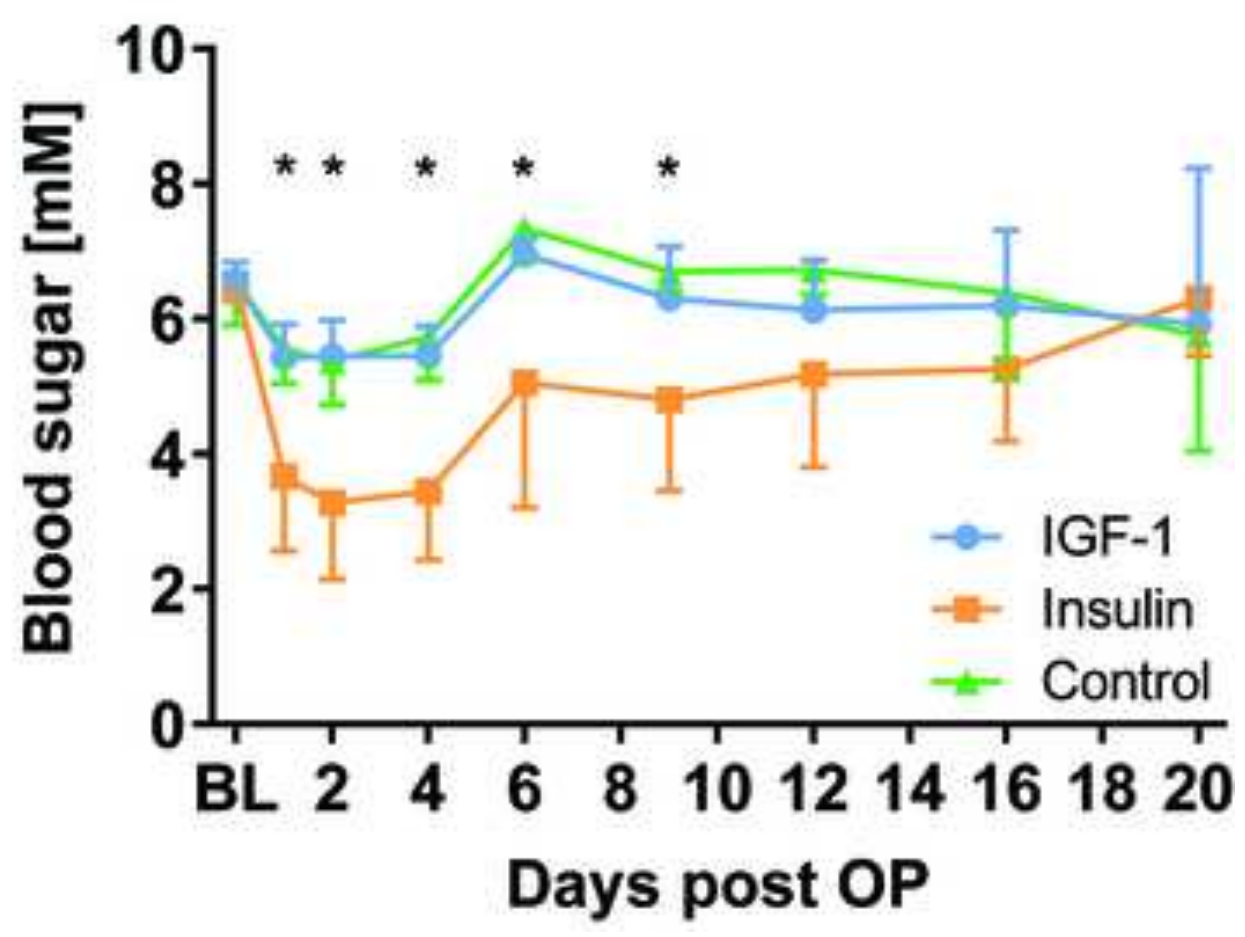



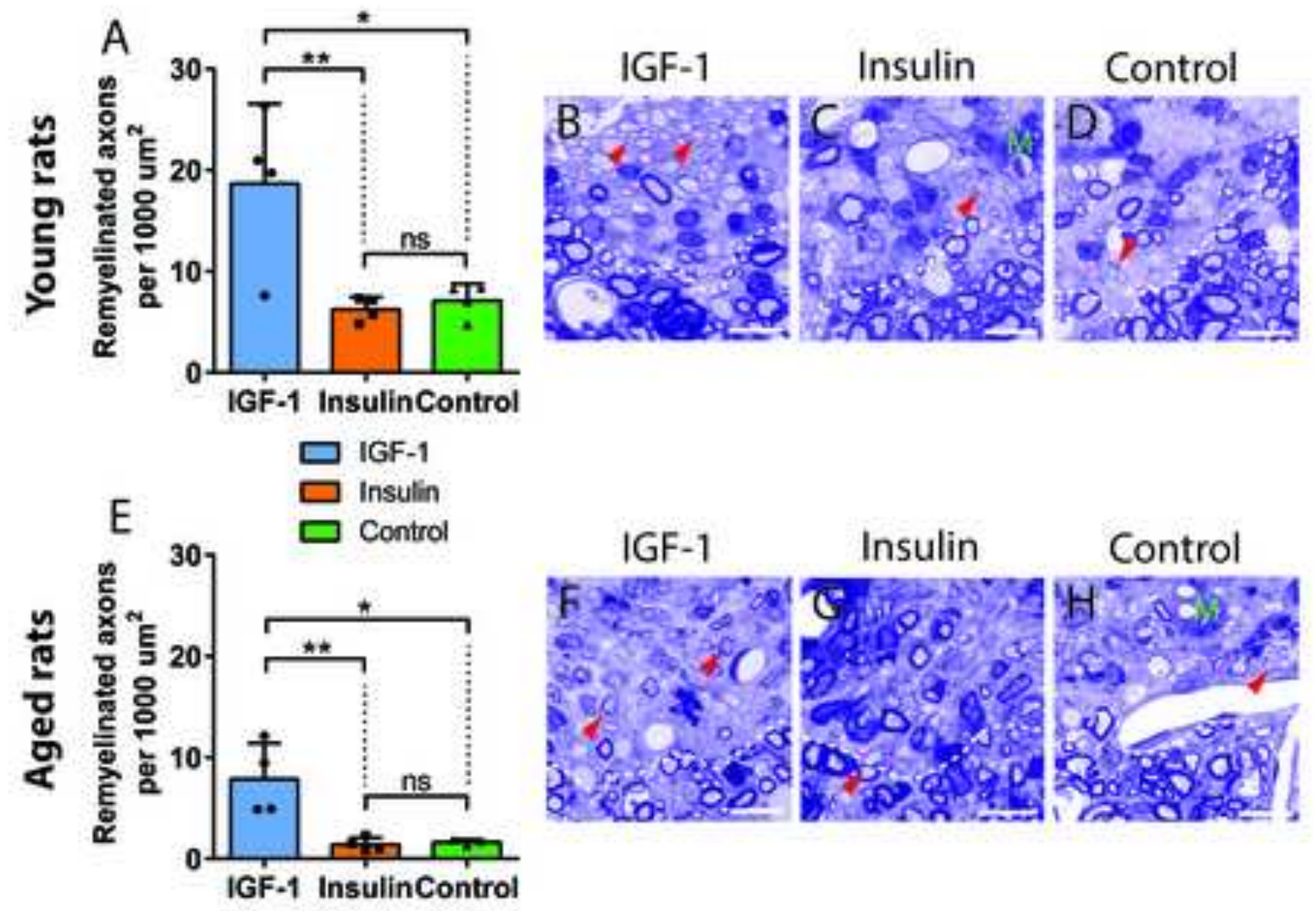
A

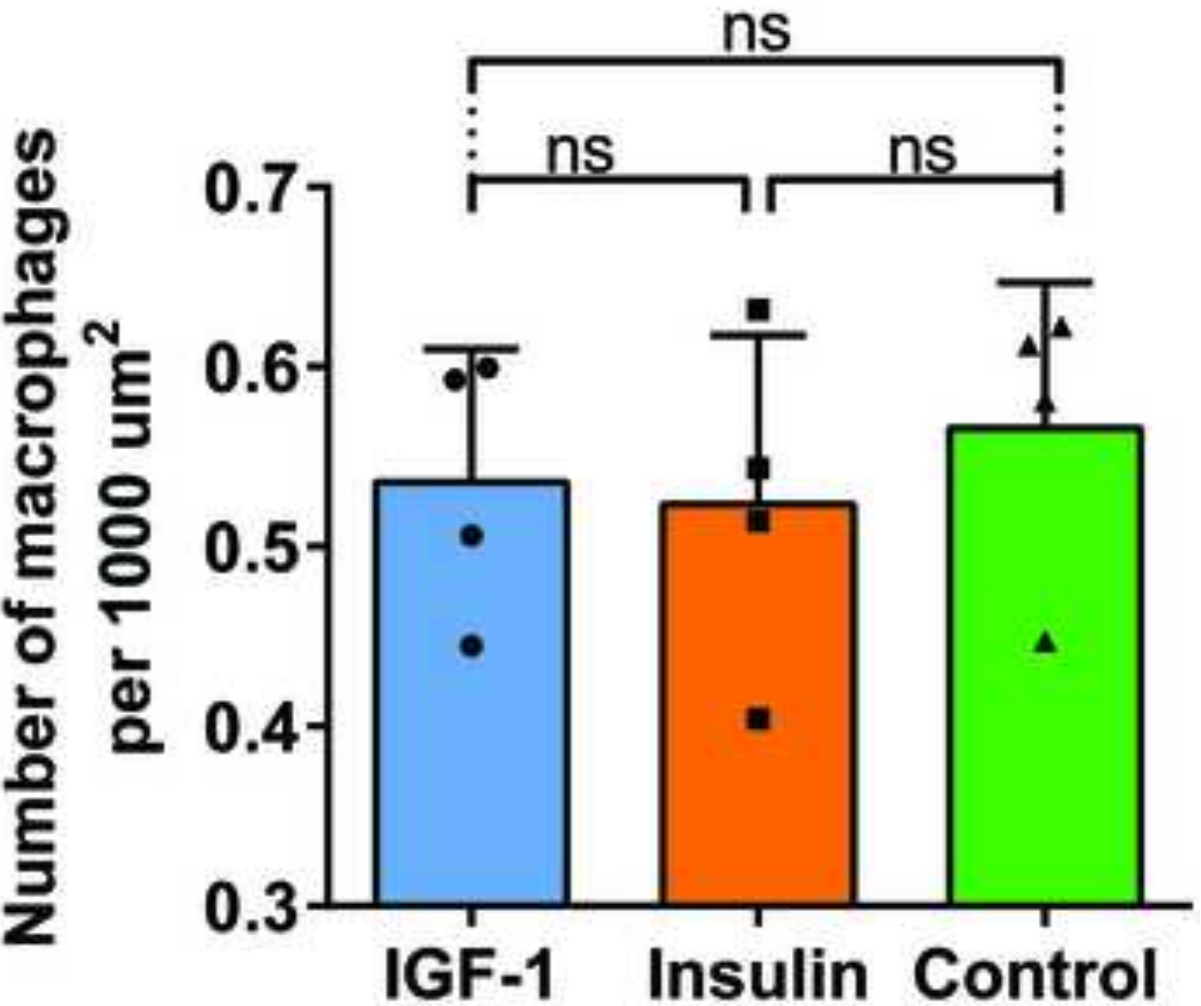

B

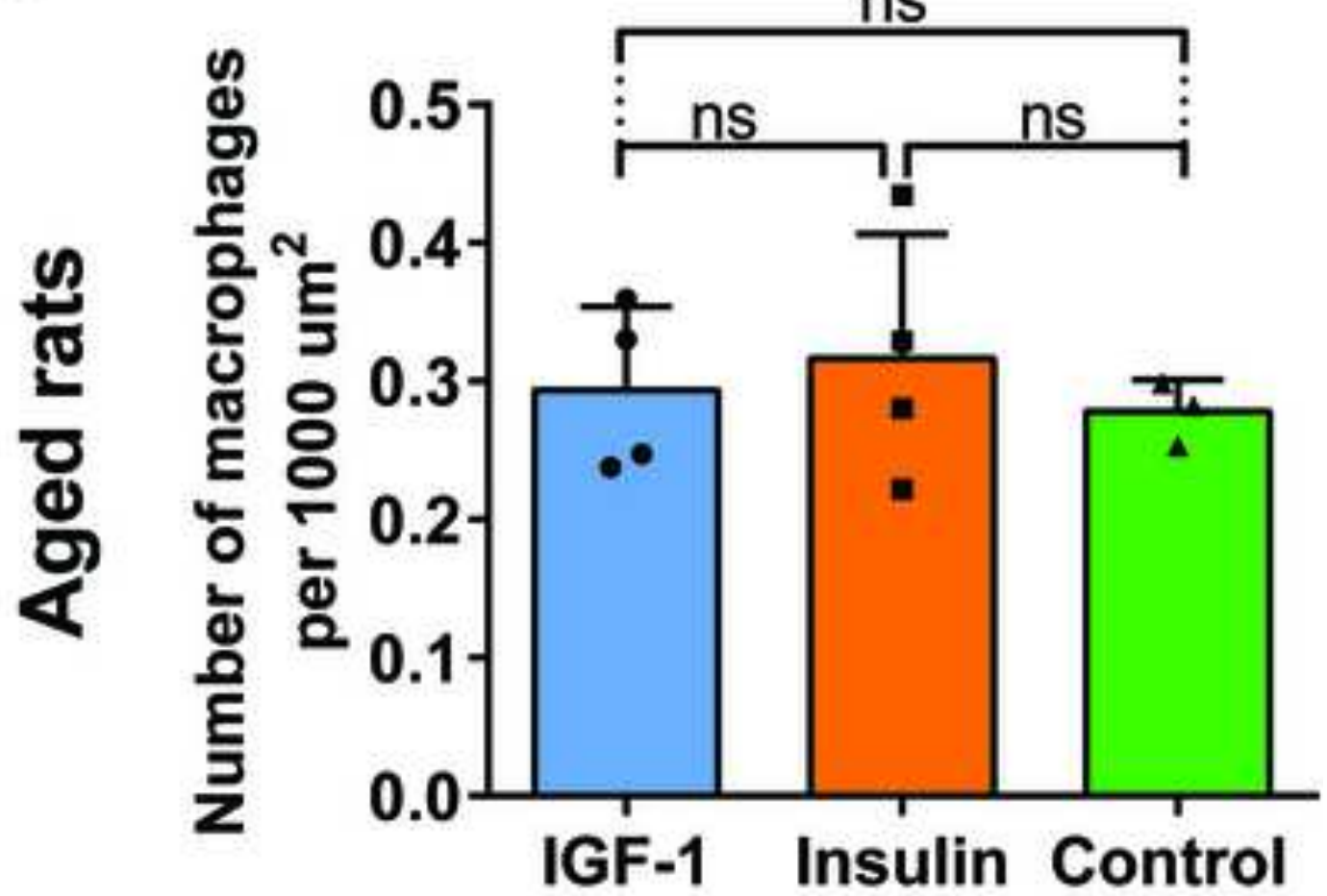

